\title{
Temporal expectancy modulates inhibition of return in a discrimination task
}

\author{
Shai Gabay and Avishai Henik \\ Ben-Gurion University of the Negev, Beer-Sheva, Israel
}

\begin{abstract}
This research examined the influence of cue temporal predictability on inhibition of return (IOR) in a discrimination task. In exogenous attention experiments, the cue that summons attention is noninformative as to where the target will appear. However, it is predictive as to when it will appear. In previous work, it was demonstrated that temporal predictability does not influence IOR in detection tasks. In this work, it is shown that IOR is influenced by temporal predictability in discrimination tasks. Predictability was manipulated by using three stimulus onset asynchrony distributions: nonaging, aging, and accelerated aging. IOR was found when the cue predicted target appearance and was modulated by temporal information. In the nonaging distribution (in which the cue did not predict target appearance), there was no IOR.
\end{abstract}

Orienting of attention can be reflexive or volitional. For example, during an amusement park mystery train ride, we might expect something to occur (temporal information), although we do not know where or what. When something does occur (for instance, a scary puppet jumping in our direction), we reflexively orient our attention toward it. Can volition modulate such reflexive/automatic processes? In the present work, we examined the influence of temporal expectancies on reflexive orienting of attention. Specifically, we manipulated the temporal information provided by a spatially nonpredictive cue.

Herein, we will start by presenting evidence for the reflexive nature of exogenous orienting of attention, continue by discussing the temporal information provided by an exogenous cue, and sum up by presenting the effect of task demands on the time course of reflexive attention.

\section{Reflexive Orienting Mechanism}

One of the ways to examine automatic orienting of attention is the spatial cuing paradigm (Posner \& Cohen, 1984). Here, a spatial nonpredictive cue is followed by a target. Responding to the target is marked by faster responding to valid trials (i.e., the target and cue appear at the same location) than to invalid trials (i.e., the target and cue appear at different locations) at short stimulus onset asynchronies (SOAs), followed by a reversal of this pattern at longer SOAs. This typical pattern of results for the later SOAs is known as inhibition of return (IOR).

IOR is considered to be a very basic and reflexive effect that enables an efficient scanning of the environment (Klein, 2000). In order to scan our environment efficiently, it is preferable not to go back to previously scanned locations but to move to new areas in the field (Klein \& MacInnes, 1999; Posner \& Cohen, 1984).
The reflexive nature of exogenous orienting is based on three sources of evidence. First, the cue in the exogenous cuing task does not predict the location of the target. For example, a cue on the right can be followed by a target on the left or on the right with the same probability. Since the cue is nonpredictive as to where the target may appear, any attentional effect generated by the cue is considered to be reflexive. The second line of evidence comes from behavioral research. Several works have demonstrated that a voluntary shift of attention does not affect IOR, which is a product of exogenous orienting (Berger, Henik, \& Rafal, 2005; Berlucchi, Chelazzi, \& Tassinari, 2000). In contrast, several researchers have suggested that capture of attention by an exogenous cue can be modulated by volitional processes (Yantis \& Jonides, 1990; for a review, see Ruz \& Lupiáñez, 2002). The third line of evidence comes from the anatomical basis of exogenous orienting. Several reports have indicated the involvement of the superior colliculus (SC) in IOR (Berger \& Henik, 2000; Rafal, Calabresi, Brennan, \& Sciolto, 1989; Ro, Shelton, Lee, \& Chang, 2004; Sapir, Soroker, Berger, \& Henik, 1999). The $\mathrm{SC}$ is a subcortical structure that is known to be involved in programming and execution of eye movements.

\section{Temporal Information Provided by an Exogenous Cue}

IOR is produced by an exogenous nonpredictive cue. In most IOR studies, although the cue does not provide any spatial information, it does provide temporal information, due to the fact that the conditional probability of the target's appearance increases with SOA. In most experiments, reaction time (RT) decreases as SOA increases. The decrease of RT with longer SOAs may indicate the participant's anticipation of the target's appearance (the foreperiod [FP] effect). Los and Agter (2005) have dem-

S. Gabay, shaigaba@bgu.ac.il 
onstrated that the relation between RT and the FP is not a result of sequential influences, which strengthens the claim that it is a volitional process.

A recent work has indicated that the spatial and temporal inhibitory processes are additive (Los, 2004). In contrast, several experiments have suggested that IOR can be modulated by manipulating temporal predictability (Milliken, Lupiáñez, Roberts, \& Stevanovski, 2003; Mondor, 1999). Recently, Tipper and Kingstone (2005) found that a high percentage of catch trials (25\%) eliminated the decline in RT as the SOA increased and reduced IOR. The authors claimed that this indicated that their participants did not use the cue as a signal to prepare for target appearance. Yet the proportion of catch trials also modulated general RT. That is, the experimental group was much slower than the control groups, which may be an indication of a change in alertness. Gabay and Henik (2008) showed that temporal predictability does not influence IOR in detection tasks and suggested that Tipper and Kingstone's results were confounded by an influence of alertness. Milliken et al. (2003) showed that temporal predictability can influence the time course of the exogenous cuing effect in discrimination tasks, but not in detection tasks. The authors manipulated the likelihood that a target would appear after a short or long cue-target interval. The discrimination task presented a larger validity effect when the target was likely to appear after a short interval. There was no such effect in the detection task. Note that there was no condition in which the cue did not predict target appearance.

\section{Task Demands}

It was originally assumed that IOR does not appear in discrimination tasks (Terry, Valdes, \& Neill, 1994), although a later work by Lupiáñez, Milan, Tornay, Madrid, and Tudela (1997) showed that the onset of IOR differed between detection and discrimination tasks. The difference in onset of IOR may be related to recruitment of different processes or amounts of attention by detection and discrimination tasks. For example, Egly and Homa (1991) suggested that detection, being a simple task, may not recruit higher processes, as the more demanding discrimination task does. Klein (2000) suggested that the difference in the time course is a product of task difficulty. In a more difficult task (discrimination), participants allocate more attention to all the stimuli (target and cue), and the longer focus of attention at the cued location delays the appearance of IOR. If, indeed, more resources are recruited for performing a more demanding task, it is possible that knowing when a target is more likely to appear is more important in a difficult discrimination task than in an easier detection task. The temporal information provided by the spatially nonpredictive cue might also influence cue processing.

\section{The Present Work}

This experiment was designed in order to examine the effects of temporal information conveyed by the cue on IOR. We manipulated temporal information by changing the frequencies of the various SOAs (see Figure 1). In the common cuing experiment, all SOAs are equally likely, so that the probability of target appearance increases as

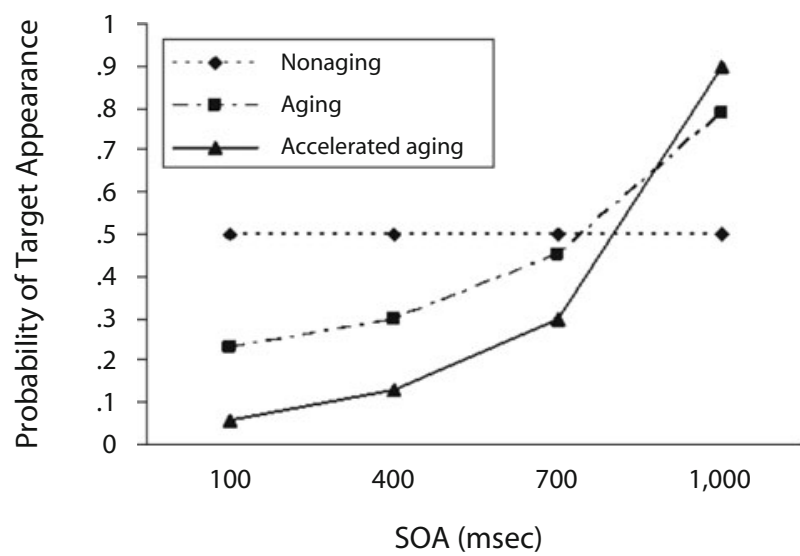

Figure 1. Changes in the probability of target appearance as a function of stimulus onset asynchrony (SOA) and trial distribution.

SOA increases. For example, in a block with four SOAs and 256 trials, 16 of which are catch trials, 60 trials would be assigned to each SOA. The probability of target appearance at the first SOA is 60 divided by the total number of trials (256), which is .23. After the first no-target SOA, the probability of target appearance at the second SOA is 60 divided by the total number of trials minus 60 (i.e., trials assigned to the first SOA), which is 196; this probability equals .3. Similarly, the probability of target appearance at the third SOA will be .44, and at the last SOA, the probability of target appearance will be .79 . This acceleration in the probability of target appearance as SOA increases (i.e., the aging design) makes the cue predictive regarding when the target will appear. In contrast, the nonaging distribution maintains a constant $50 \%$ chance that the target will appear at any given SOA. In the present experiment, the first SOA was assigned on half of the trials (128), so that the probability of target appearance was .5. The second SOA was assigned on half of the remaining trials (64), so that the probability of target appearance remained .5, and so on for the third and fourth SOAs. This constant probability of target appearance throughout the different SOAs makes the cue noninformative as to when the target will appear. In addition to the nonaging condition, we used an accelerated-aging condition, which was the reverse of the nonaging condition (Baumeister \& Joubert, 1969). This condition is characterized by an accelerated probability of target appearance as SOA increases. At the first SOA, target probability was .06; at the second SOA, it was .13; at the third, .3; and at the fourth, .9. Hence, this arrangement made the cue more informative as to when the target would appear, as compared with the other conditions.

Näätänen (1970) has shown that by using a nonaging foreperiod design, the effect of the decline in RT with foreperiod increase can be eliminated. This finding can be attributed to the disappearance of the participants' expectancy as to when the target will appear. The present design enabled examining whether expectancy, a volitional process (Los \& Agter, 2005), could influence IOR, a reflexive-automatic phenomenon. In our work, the nonaging condition cue did not convey any temporal or spatial 
information. In contrast to previous work (Milliken et al., 2003), this enabled us to examine the influence of temporal predictability in a discrimination task by comparing a temporally nonpredictive (nonaging) cue with a temporally predictive (aging or accelerated-aging) cue.

\section{METHOD}

The experimental procedure for this experiment was based on the work of Gabay and Henik (2008) in order to enable a comparison of the influence of temporal predictability in detection versus discrimination tasks.

\section{Participants}

Forty-two participants from Ben-Gurion University of the Negev participated in the experiment in exchange for course credit. The participants were randomly assigned to one of three experimental groups: aging FP, nonaging FP, or accelerated-aging FP.

\section{Apparatus and Stimuli}

The stimuli were white figures on a black background, consisting of a fixation $\operatorname{dot}\left(0.3^{\circ}\right)$ at the center of the computer screen and three square boxes $\left(2.5^{\circ}\right.$ each side $)$, one at the center of the screen and the other two $6.5^{\circ}$ from the center of the screen. A target letter "O" or "Q" $\left(1.3^{\circ}\right)$ appeared in the center of one of the peripheral boxes. The target letter was preceded by a brightening of one of the two peripheral boxes, which was accomplished by widening the box's contour from 1 to $5 \mathrm{~mm}$. The participants responded to the target letter by pressing one of two buttons on a response box. The appropriate response was counterbalanced between participants.

\section{Procedure}

The participants were tested in a dimly illuminated room. They were seated $57 \mathrm{~cm}$ from the computer monitor. The participants were instructed to maintain fixation on the fixation point throughout the experiment. The experimenter monitored the participants' eye movements through a JVC-TK240 video camera. If an eye movement was detected, the experimenter sent an alerting sound to the participant. The participants were instructed to press one of two possible buttons as quickly as possible when the target letter appeared but to avoid false responses. The assignment of the letters to the response buttons was counterbalanced across participants. The participants were also informed that the peripheral cue was not informative as to where the target would appear. Each trial began with the appearance of the fixation dot for $500 \mathrm{msec}$. Four hundred milliseconds after the disappearance of fixation, one of two peripheral boxes brightened for $75 \mathrm{msec}$ (the cue). The target letter appeared in one of the peripheral boxes $100,400,700$, or $1,000 \mathrm{msec}$ after the onset of the cue and remained in view until the participant responded. On $6 \%$ of the trials, no target appeared (i.e., catch trials), and the participant was instructed not to respond. Each participant had eight practice trials before the experiment began. The participants were assigned to one of three experimental groups: (1) an aging FP group, in which the different SOAs were equally likely; (2) a nonaging FP group, in which, for all SOAs, there was a $50 \%$ chance that the target would appear; and (3) an accelerated-aging FP group, in which there was a reversed distribution of SOAs from that of the nonaging FP group.

The experiment was divided into four blocks of 256 trials (i.e., a total of 1,024 trials per participant).

\section{RESULTS}

Trials on which the participants responded incorrectly $(3.3 \%$ of the data), very quickly $(\leq 100 \mathrm{msec})$, or very slowly ( $\geq 1,000 \mathrm{msec}$, less than $2 \%$ ) were excluded from the analysis. No false response was made during the catch trials. RTs as a function of cue validity and SOA for every experimental group are presented in Figure 2.

As can be seen, the pattern of results fits the hypothesis that SOA distribution can influence the expectancy of participants. In the nonaging FP group, RT has a positive slope - it increases as SOA increases-whereas in the aging FP group and the accelerated-aging group, RTs decrease as SOAs increase. An ANOVA with group (nonaging, aging, accelerated aging), SOA (100, 400, 700, or 1,000 msec), and validity (valid, invalid) as factors confirmed this observation. There was a significant main effect of SOA $\left[F(3,117)=4.2, p<.01, \eta_{\mathrm{p}}^{2}=.09\right]$ and of validity $\left[F(1,39)=13.97, p<.001, \eta_{\mathrm{p}}^{2}=.26\right]$. The SOA $\times$ group interaction was significant $[F(6,117)=16.2, p<$ $\left..001, \eta_{\mathrm{p}}^{2}=.45\right]$. This interaction was further analyzed through an interaction between comparisons that examined the nonaging group linear trend with the aging group linear trend. This comparison was significant $[F(1,39)=$ $\left.13.28, p<.001, \eta_{\mathrm{p}}^{2}=.2\right]$. A comparison of the linear trend of the aging and the accelerated-aging groups also produced a significant effect $[F(1,39)=18.5, p<.001$, $\left.\eta_{\mathrm{p}}^{2}=.23\right]$. These results indicate that the experimental manipulation did influence the participants' expectancy. The SOA $\times$ validity and validity $\times$ group interactions were also significant $\left[F(3,117)=11.7, p<.001, \eta_{\mathrm{p}}^{2}=.23\right.$, and $F(2,39)=9.5, p<.001, \eta_{\mathrm{p}}^{2}=.32$, respectively $]$.

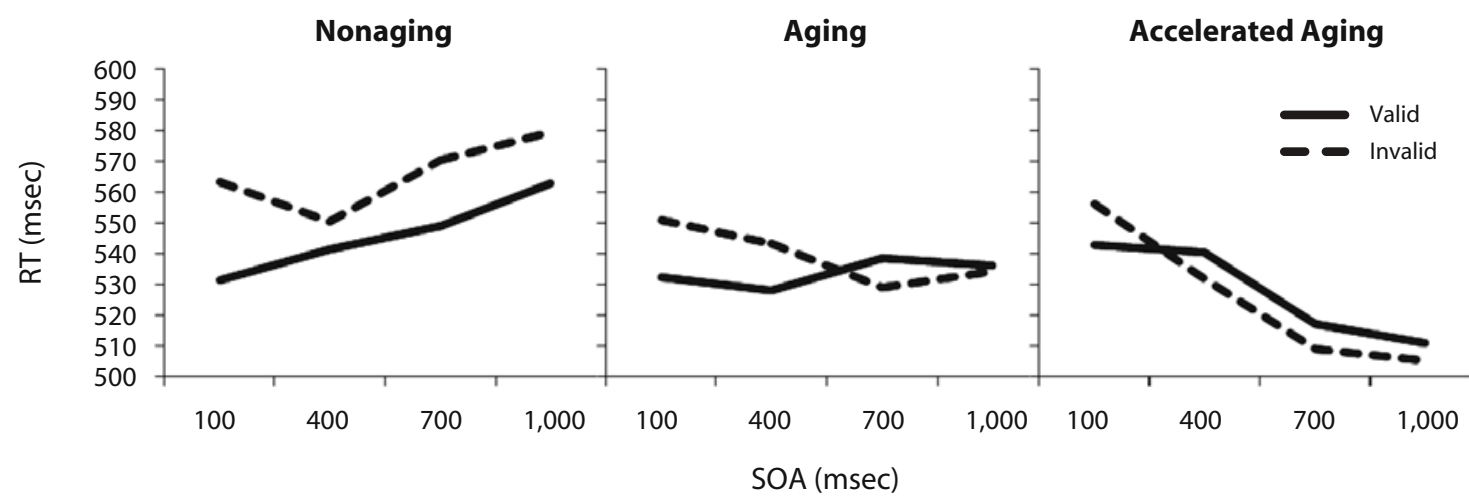

Figure 2. Reaction times (RTs) as a function of stimulus onset asynchrony (SOA), validity, and group. 
The triple SOA $\times$ validity $\times$ group interaction was significant $\left[F(6,117)=2.88, p<.05, \eta_{\mathrm{p}}^{2}=.12\right]$. As can be seen in Figure 2, IOR was not apparent in the nonaging group, appeared at the third SOA in the aging group, and appeared at the second SOA in the accelerated-aging group. We further analyzed the triple interaction in order to determine whether the time course of the IOR effect was modulated by group. A comparison of the validity effect between the nonaging and the aging groups for the different SOAs $\left[F(1,39)=2.5, p=.12, \eta_{\mathrm{p}}^{2}=.13 ; F(1,39)<1\right.$, $p=.4, \eta_{\mathrm{p}}^{2}=.02 ; F(1,39)=23.5, p<.001, \eta_{\mathrm{p}}^{2}=.43$; $F(1,39)=4.2, p<.05, \eta_{\mathrm{p}}^{2}=.01 ;$ for $100-, 400-, 700-$, and 1,000 -msec SOAs, respectively] indicated a difference in the validity effect between the groups from the third SOA on. A comparison of the validity effect between the aging and the accelerated-aging groups for the different SOAs $\left[F(1,39)<1, p=.49, \eta_{\mathrm{p}}^{2}=.01 ; F(1,39)=11, p<.01\right.$, $\eta_{\mathrm{p}}^{2}=.28 ; F(1,39)<1, p=.85, \eta_{\mathrm{p}}^{2}=.001 ; F(1,39)<1$, $p=.68, \eta_{\mathrm{p}}^{2}=.03$; for 100-, 400-, 700-, and 1,000-msec SOAs, respectively] indicated that the validity effect was significantly different between the groups only at the second SOA. Those comparisons indicate that the time course of IOR was accelerated for the accelerated-aging group, as compared with the aging group, and that no IOR was apparent for the nonaging group.

In order to demonstrate that a significant validity effect and a later IOR were observed in the aging and acceleratedaging groups, we conducted planned comparisons of the simple validity effects at each SOA. The comparisons for the aging group indicated that a robust validity effect was observed for the 100- and 400-msec SOAs and that a significant IOR was observed at the 700-msec SOA $[F(1,13)=$ $17.26, p<.01, \eta_{\mathrm{p}}^{2}=.57 ; F(1,13)=7.45, p<.05, \eta_{\mathrm{p}}^{2}=$ $.36 ; F(1,13)=6.53, p<.05, \eta_{\mathrm{p}}^{2}=.33 ; F(1,13)<1, p=$ $.48, \eta_{\mathrm{p}}^{2}=.03$, for $100-, 400-, 700-$, and $1,000-\mathrm{msec}$ SOAs, respectively]. The same comparisons for the acceleratedaging group indicated that no validity effect was observed, and that a significant IOR was observed at the 700 -msec SOA and a marginally significant IOR was observed at the $1,000-\mathrm{msec}$ SOA $\left[F(1,13)=3.18, p=.09, \eta_{\mathrm{p}}^{2}=.19\right.$; $F(1,13)=3, p=.1, \eta_{\mathrm{p}}^{2}=.18 ; F(1,13)=5, p<.05, \eta_{\mathrm{p}}^{2}=$ .27 ; and $F(1,13)=4.1, p=.06, \eta_{\mathrm{p}}^{2}=.24$, for $100-, 400-$, 700-, and 1,000-msec SOAs, respectively].

We also conducted an ANOVA for error percentage. The only significant effect was for $\operatorname{SOA}[F(3,117)=3.16$, $\left.p<.05, \eta_{\mathrm{p}}^{2}=.07\right]$, which indicated an increase in error percentage as the SOA became longer. An examination of the different trends revealed only a significant linear trend $\left[F(1,36)=7.04, p<.001, \eta_{\mathrm{p}}^{2}=.48\right]$.

\section{DISCUSSION}

In this experiment, we demonstrated that the time course of exogenous attentional orienting is influenced by temporal predictability. In the nonaging group condition, which did not contain temporal information, IOR was not apparent even after 1,000 msec. In the accelerated-aging group condition, which contained the highest temporal information, the shift from facilitation to IOR appeared earlier, in comparison with the aging group condition.
The influence of volition on reflexive and automatic processes is an intriguing issue. In the present work, we systematically examined the influence of temporal expectancy on the time course of IOR. We demonstrated that as the cue provided more temporal information, appearance of IOR was accelerated. When no temporal information was provided by the cue, no IOR was apparent.

The difference between detection and discrimination tasks initiated experimental work in the area of attention. Early works (Terry et al., 1994) suggested that IOR was eliminated in discrimination tasks. In contrast, further work showed that IOR may appear in discrimination tasks later than in detection tasks (Lupiáñez et al., 1997). Egly and Homa (1991) suggested that detection, being a simple task, may not recruit the higher processes that the more demanding discrimination task does. It is possible that the more demanding tasks are more susceptible to temporal information. A mechanism that might be involved in the difference between the tasks is the locuscoeruleus-norepinephrine system (LC-NE). Aston-Jones and Cohen (2005) presented a theory that suggested two different modes of activity - tonic and phasic — of the LC. The shift between the two modes is guided by brain areas that are involved in monitoring task-related utility and external feedback (the anterior cingulate and orbitofrontal cortices). Participants are more likely to use the phasic LC mode when external feedback is present and higher accuracy is needed (as in a discrimination task).

We propose two possible accounts for the time course modulation of the attentional effect in discrimination tasks. The first concerns the influence of temporal expectancy on cue processing, and the second suggests a general influence of expectancy on the time course of attentional effects. As was suggested in the introduction, in a more demanding task, the ability to predict target appearance is more desired. A cue that provides temporal information might be processed more quickly than a temporally nonpredictive cue, since it conveys critical information regarding the appearance of the target. Faster processing of the cue, when it conveys more temporal information, would explain the difference in the time course of the attentional effect. IOR was presented earliest when the cue conveyed the most temporal information (accelerated-aging condition) and later in the less predictive condition (aging condition). Whether IOR was completely abolished in the nonaging condition or just substantially delayed should be examined in future research. The second possible explanation suggests a general influence of expectancy on the time course of attentional effects. In a recent work, Chica, Lupiáñez, and Bartolomeo (2006) demonstrated that in a discrimination task, IOR was presented earlier in expected locations (at which the target was more likely to appear) than in unexpected locations. This finding, in conjunction with the present work, might indicate that expectancy, whether temporal or spatial, accelerates the time course of exogenous orienting in discrimination tasks, but not in detection tasks.

The influence of task demands and volitional processes on reflexive attentional effects is an ongoing research interest. Future work will surely provide insights about the ability of volitional processes to modulate reflexive ones. 


\section{AUTHOR NOTE}

Correspondence concerning this article should be addressed to S. Gabay, Department of Psychology, Ben-Gurion University of the Negev, P.O. Box 653, Beer-Sheva, Israel 84105 (e-mail: shaigaba@bgu .ac.il).

\section{REFERENCES}

Aston-Jones, G., \& CohEn, J. D. (2005). An integrative theory of locus coeruleus-norepinephrine function: Adaptive gain and optimal performance. Annual Review of Neuroscience, 28, 403-450. doi:10.1146/ annurev.neuro.28.061604.135709

Baumeister, A. A., \& Joubert, C. E. (1969). Interactive effects on reaction time of preparatory interval length and preparatory interval frequency. Journal of Experimental Psychology, 82, 393-395. doi:10.1037/h0028119

BERger, A., \& HENIK, A. (2000). The endogenous modulation of IOR is nasal-temporal asymmetric. Journal of Cognitive Neuroscience, 12, 421-428. doi:10.1162/089892900562246

Berger, A., Henik, A., \& Rafal, R. (2005). Competition between endogenous and exogenous orienting of visual attention. Journal of Experimental Psychology: General, 134, 207-221. doi:10.1037/0096 $-3445.134 .2 .207$

Berlucchi, G., Chelazzi, L., \& Tassinari, G. (2000). Volitional covert orienting to a peripheral cue does not suppress cue-induced inhibition of return. Journal of Cognitive Neuroscience, 12, 648-663. doi:10.1162/089892900562408

Chica, A., Lupiáñez, J., \& Bartolomeo, P. (2006). Dissociating inhibition of return from endogenous orienting of spatial attention: Evidence from detection and discrimination tasks. Cognitive Neuropsychology, 23, 1015-1034. doi:10.1080/02643290600588277

EgLY, R., \& HoмA, D. (1991). Reallocation of visual attention. Journal of Experimental Psychology: Human Perception \& Performance, 17, 142-159. doi:10.1037/0096-1523.17.1.142

Gabay, S., \& HenIK, A. (2008). The effects of expectancy on inhibition of return. Cognition, 106, 1478-1486. doi:10.1016/j.cognition.2007 .05 .007

KLEIN, R. (2000). Inhibition of return. Trends in Cognitive Sciences, 4 , 138-146. doi:10.1016/S1364-6613(00)01452-2

KLeIN, R., \& MACInNES, W. J. (1999). Inhibition of return is a foraging facilitator in visual search. Psychological Science, 10, 346-352. doi:10.1111/1467-9280.00166

Los, S. A. (2004). Inhibition of return and nonspecific preparation: Separable inhibitory control mechanisms in space and time. Perception \& Psychophysics, 66, 119-130.
Los, S. A., \& Agter, F. (2005). Reweighting sequential effects across different distributions of foreperiods: Segregating elementary contributions to nonspecific preparation. Perception \& Psychophysics, 67, 1161-1170.

Lupiáñez, J., Milan, E. G., Tornay, F. J., Madrid, E., \& Tudela, P. (1997). Does IOR occur in discrimination tasks? Yes, it does, but later. Perception \& Psychophysics, 59, 1241-1254.

Milliken, B., Lupiáñez, J., Roberts, M., \& Stevanovski, B. (2003). Orienting in space and time: Joint contributions to exogenous spatial cuing effects. Psychonomic Bulletin \& Review, 10, 877-883.

Mondor, T. A. (1999). Predictability of the cue-target relation and the time-course of auditory inhibition of return. Perception \& Psychophysics, 61, 1501-1509.

NÄÄTÄNEN, R. (1970). The diminishing time-uncertainty with the lapse of time after the warning signal in reaction-time experiments with varying fore-periods. Acta Psychologica, 34, 399-419.

Posner, M. I., \& Cohen, Y. P. C. (1984). Components of visual orienting. In H. Bouma \& D. G. Bouwhuis (Eds.), Attention and performance X: Control of language processes (pp. 531-556). Hillsdale, NJ: Erlbaum.

Rafal, R., Calabresi, P. A., Brennan, C. W., \& Sciolto, T. K. (1989). Saccade preparation inhibits reorienting to recently attended locations. Journal of Experimental Psychology: Human Perception \& Performance, 15, 673-685. doi:10.1037/0096-1523.15.4.673

Ro, T., Shelton, D., Lee, O. L., \& Chang, E. (2004). Extrageniculate mediation of unconscious vision in transcranial magnetic stimulationinduced blindsight. Proceedings of the National Academy of Sciences, 101, 9933-9935.

Ruz, M., \& LuPiáñEz, J. (2002). A review of attentional capture: On its automaticity and sensitivity to endogenous control. Psicologica, 23, 283-309.

SAPIr, A., Soroker, N., Berger, A., \& HeniK, A. (1999). Inhibition of return in spatial attention: Direct evidence for collicular generation. Nature Neuroscience, 2, 1053-1054. doi:10.1038/15977

Terry, K. M., Valdes, L. A., \& NeILl, W. T. (1994). Does "inhibition of return" occur in discrimination tasks? Perception \& Psychophysics, $\mathbf{5 5}, 279-286$

Tipper, C., \& Kingstone, A. (2005). Is inhibition of return a reflexive effect? Cognition, 97, B55-B62. doi:10.1016/j.cognition.2005.02.003

Yantis, S., \& Jonides, J. (1990). Abrupt visual onsets and selective attention: Voluntary versus automatic allocation. Journal of Experimental Psychology: Human Perception \& Performance, 16, 121-134. doi:10.1037/0096-1523.16.1.121

(Manuscript received May 31, 2009; revision accepted for publication October 20,2009.) 